\title{
Expression of Phospholipase D Isozymes in Mouse Lungs during Postnatal Development
}

\author{
Changjong MOON ${ }^{1) * *}$, Jinwoo JEONG ${ }^{2) * *}$, Min Kyoung $\mathrm{SHIN}^{3)}$, Do Sik $\mathrm{MIN}^{3)}$ and Taekyun $\mathrm{SHIN}^{2}$ * \\ ${ }^{1)}$ Department of Veterinary Anatomy, College of Veterinary Medicine and Animal Medical Institute, Chonnam National University, \\ Gwangju 500-757, ${ }^{2)}$ Department of Veterinary Anatomy, College of Veterinary Medicine and Applied Radiological Science Research \\ Institute, Jeju National University, Jeju 690-756 and ${ }^{3)}$ Department of Molecular Biology, College of Natural Science, Pusan National \\ University, Busan 609-735, South Korea
}

(Received 18 December 2008/Accepted 3 February 2009)

ABSTRACT. The expression level of phospholipase D (PLD) was examined in the lungs of mice during postnatal development. Immunoprecipitation analysis revealed a moderate basal level of PLD1 in the lungs at postnatal 1 week, with a gradual increase occurring until week 4. The pattern of PLD2 paralleled that of PLD1, although the quantity of PLD2 was markedly less. PLD was immunostained in the most bronchiolar epithelium, some pulmonary macrophages and alveolar cells at postnatal week 1, with increased immunoreactivity in those cells with lung development at postnatal week 4 . These findings suggest that the increase of PLD expression is associated with pulmonary alveolarization, possibly by involvement in cell proliferation and differentiation during postnatal mouse lung development. KEY WORDS: alveolarization, lung, mouse, phospholipase D, postnatal development.

Phospholipase D (PLD) is ubiquitously present in all mammalian cells, and is one of major membrane phospholipids that forms phosphatidic acid (PA) and choline [7]. PLD is involved in multiple physiological processes including cell proliferation, differentiation, secretion, and migration [3, 7]. PLD isozymes including both PLD1 and 2 are expressed in a variety of normal mouse tissues including the lungs [11]. PLD expression is involved in cell differentiation during postnatal organogenesis in the brain [12], testis [13], hearts [16] and retina [14].

The organogenesis of the lung is achieved by branching of the conducting airways and by septation to subdivide the airspaces (alveolarization) [1]. The development of lungs after birth has been characterized in mice [2]. Lung postnatal development is regulated by many factors including transcription factors, peptide growth factors and physical forces [5]. PLD is an important enzyme involved in the development of organs after birth such as the lungs; the latter markedly increase in volume within a few weeks following birth. In pulmonary epithelial and vascular endothelial cells, PLD can be stimulated by a wide variety of agonists including neurotransmitters, growth factors, hormones, antibodies, bioactive lipids, calcium ionophores, phorbol esters and reactive oxygen species (ROS) [6]. However, little is known concerning how the expression of PLD is regulated during postnatal development in the lungs of laboratory animals including mice. The aims of this study were to examine the expression and cellular localization of PLD in lungs of mice during postnatal development using Western blot analysis and immunohistochemistry.

\footnotetext{
* Correspondence to: Shin, T., Department of Veterinary Anatomy, College of Veterinary Medicine, Jeju National University, Aradong, Jeju 690-756, South Korea.

e-mail: shint@jejunu.ac.kr

**The first two authors equally contributed to this work.
}

BALB/c adult mice (Orient Bio, Gyunggi-do, Korea) of both genders were maintained and mated in our laboratory. Lung tissues (six samples at each time point) from 2-3 litters were sampled at 1, 2, 4 and 8 weeks after birth, and examined using Western blotting and histological analysis. All animal experiments followed a protocol approved by the Committee for Animal Experimentation at the Jeju National University.

For the Western blot analysis, lung tissues were dissected and lysed in immunoprecipitation buffer [20 mM HEPES (pH 7.2), 1\% Triton X-100, $1 \%$ deoxycholate, $0.1 \%$ sodium dodecyl sulfate (SDS), $150 \mathrm{mM} \mathrm{NaCl}, 10 \mu \mathrm{g} / \mathrm{m} l$ leupeptin, $10 \mu \mathrm{g} / \mathrm{m} l$ aprotinin and $1 \mathrm{mM}$ phenylmethylsulfonylfluoride, $\mathrm{pH}$ 7.4]. Pieces of lung were fixed in 4\% paraformaldehyde in phosphate buffer and embedded in paraffin. Paraffin sections were stained with hematoxylin and eosin, and these sections were also used for immunohistochemistry.

Immunoprecipitation and Western blot analyses were done as previously described [11]. In brief, the lysate supernatant of lung tissues was pre-cleared by incubation with preimmune IgG and protein A-Sepharose for $30 \mathrm{~min}$. Precleared cell lysates were incubated with the anti-PLD antibody and $30 \mu l$ of the $50 \%$ slurry of protein A-Sepharose for $4 \mathrm{hr}$. The immune complex was collected by centrifugation and washed five times with ice-cold buffer that contained 20 $\mathrm{mM}$ Tris (pH 7.5), $1 \mathrm{mM}$ EDTA, $1 \mathrm{mM}$ EGTA, $150 \mathrm{mM}$ $\mathrm{NaCl}, 2 \mathrm{mM} \mathrm{Na}_{3} \mathrm{VO}_{4}, 10 \%$ glycerol and 1\% Nonidet P-40. SDS sample buffer was added and the mixture was boiled. The recovered proteins were separated by SDS-polyacrylamide gel electrophoresis. The separated proteins were transferred to an Immobilon-P polyvinylidene difluoride membrane (Millipore, Billerica, MA, U.S.A.), blocked in $5 \%$ non-fat dried milk and probed with anti-PLD antibody as indicated. Immunoreactive bands were visualized by 
chemiluminescence using horseradish peroxidase-conjugated secondary antibodies and the enhanced chemiluminescence reagents (Amersham Biosciences, Piscataway, NJ, U.S.A.).

Paraffin sections (5- $\mu \mathrm{m}$ thickness) were deparaffinized and pretreated with $0.01 \mathrm{M}$ citrate buffer $(\mathrm{pH}$ 6.0) in a microwave oven for $3 \mathrm{~min}$. After hydration, the sections were treated with $0.3 \%$ hydrogen peroxide in distilled water for $20 \mathrm{~min}$ to block endogenous peroxidase activity. Following three washes in phosphate buffered saline, the sections were exposed to $10 \%$ normal goat serum and allowed to react with the affinity-purified anti-PLD antibody. Immunoreactivity was visualized with the avidin-biotin peroxidase reaction (Vector Elite ABC kit; Vector Labs, Burlingame, CA, U.S.A.). Peroxidase was developed with diaminobenzidine substrate kit (Vector Labs). The sections were counterstained with hematoxylin before being mounted.

To visualize the co-localization of PLD and isolectin B4 (IB4) in the mouse lung tissues, the sections were reacted with biotinylated IB4 (Sigma-Aldrich, St. Louis, MO, U.S.A.), followed by tetramethylrhodaminoisothiocyanate (TRITC)-labeled streptavidin (Zymed Laboratories, San Francisco, CA, U.S.A.). Biotinylated IB4 derived from Griffonia simplicifolia (Sigma-Aldrich) was used to label macrophages and mucous-secreting epithelial cells [10], as IB4 has a strong affinity for terminal $\alpha \mathrm{D}$-galactosyl residues that are abundant in macrophages [9] and some epithelial cells [8]. The sections were then reacted with the anti-PLD antibody, followed by fluorescein isothiocyanate (FITC)labeled goat anti-rat IgG (Zymed Laboratories).

As shown in Fig. 1, the protein level of PLD isozymes increased during postnatal development of lungs. The PLD1 level was significantly detected at 1 week after birth, slightly increased until 2 weeks postnatally, and then significantly increased at 4 weeks after birth. At postnatal week 8 , the level of PLD1 expression was significantly declined to the level at postnatal 1 week. In contrast to the level of PLD1, PLD2 protein was negligibly detected at 1 week postnatally and then moderately increased. After postnatal weeks 8 , the expression level of PLD2 declined to the basal level.

We designated PLD rather than each PLD1 or PLD2 in immunohistochemical study, because anti-PLD antibody used in this study recognizes both PLD1 and PLD2 as shown in Fig. 1 and our previous study [11]. At postnatal week 1, PLD was localized in the covering epithelia of the bronchioles and some round cells, as well as in pneumocytes of the alveoli (Fig. 2A). A similar finding was also confirmed in lungs at 2 weeks postnatally (Fig. 2B). At 4 weeks after birth, immunoreactivity of PLD was detected in bronchiolar epithelia and pneumocytes of the alveoli (Fig. 2C). The intensity of PLD immunoreactivity in the lungs was enhanced with development from 1-4 weeks after birth, matching the result of Western blot analysis (Fig. 1).

To observe the cell phenotype of PLD in lungs, double labeling was performed using IB4 lectin to visualize macrophages and mucous-secreting epithelial cells. PLD-posi-
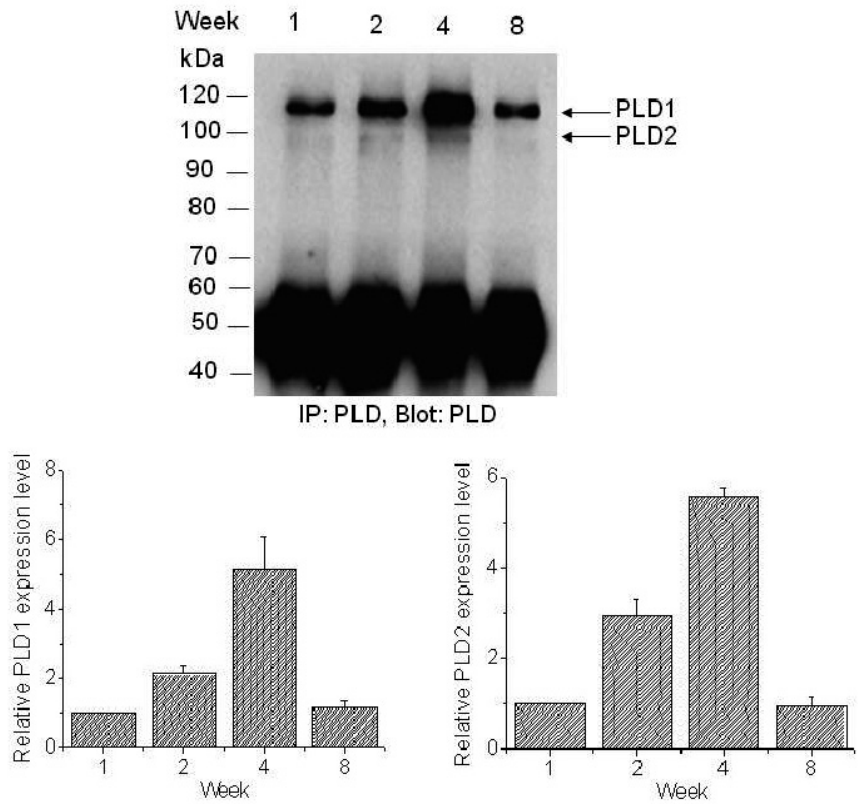

Fig. 1. Western blot analysis of PLD1 and PLD2 expression in mouse lungs. Tissue homogenates were immunoprecipitated and immunoblotted with anti-PLD antibody, which specifically recognizes PLD1 and PLD2 in mouse lung. The line on the left indicates the molecular size marker. The relative expression level of PLD isozymes was quantified using a densitometer. 

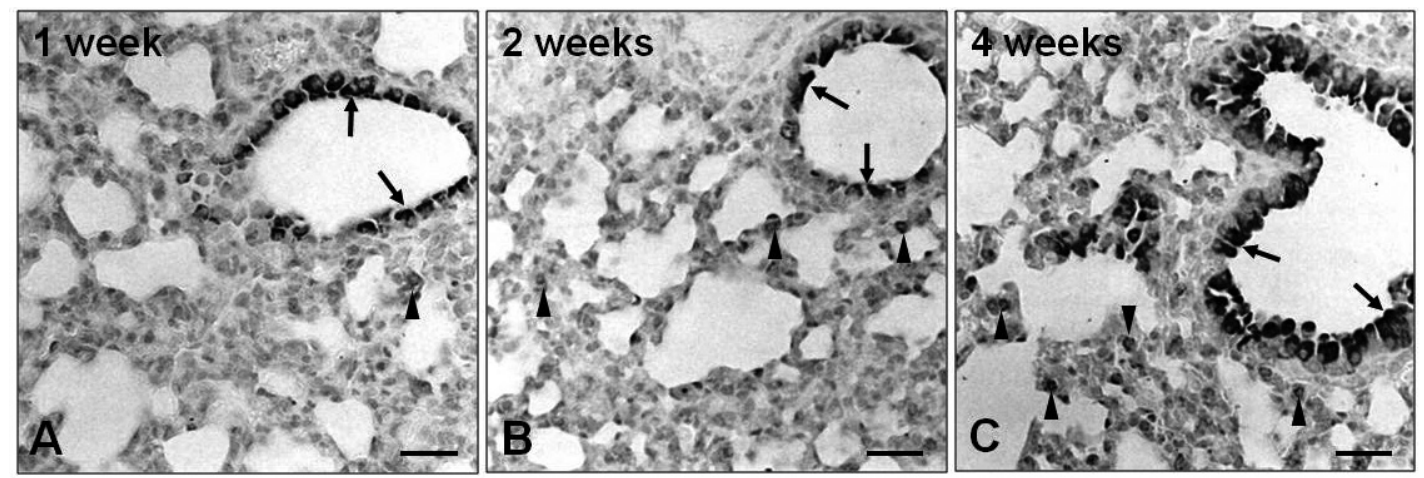

Fig. 2. Immunohistochemical localization of PLD in the lungs at 1 (A), 2 (B), and 4 (C) weeks after birth. In the lungs at all ages, PLD is localized in the covering epithelia of the bronchioles (arrows) and some macrophages, as well as in pneumocytes of the alveoli (arrowheads). Counterstained with hematoxylin. All scale bars represent $30 \mu \mathrm{m}$.
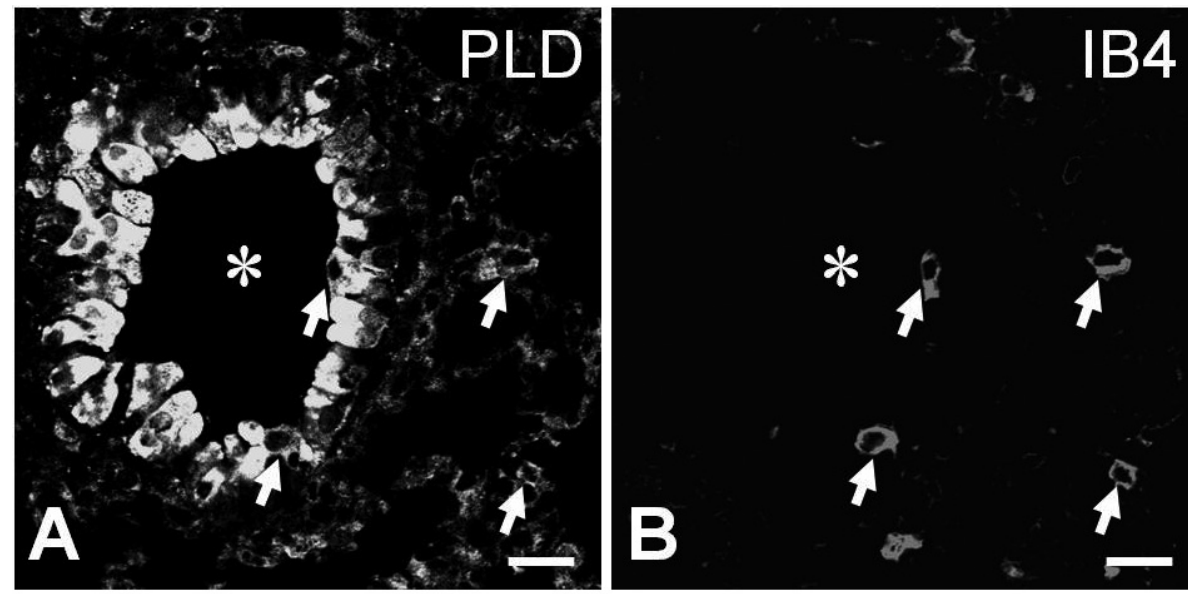

Fig. 3. Co-localization of PLD and IB4 in lungs of mouse (4 weeks). Lungs were stained with PLD antibody (A) and biotinylated IB4 (B). Asterisks indicate bronchiolar space. The immunoreactivity of PLD is detected in IB4-positive bronchiolar epithelial cells and/or alveolar macrophages (A and $\mathrm{B}$, arrows). Scale bars represent $20 \mu \mathrm{m}$

tive cells (Fig. 3A) were co-localized with IB4 reactivity (Fig. 3B) in some epithelial cells in the bronchiole and macrophages in the alveoli.

PLD is transiently upregulated with postnatal development of cerebrum [12], cerebellum [12], hippocampus [15], retina [14], heart [16] and testis [13] in laboratory animals including rats and mice. It has been suggested that PLD is associated with cell proliferation and differentiation in postnatal development $[3,7]$.

Lungs develop markedly after birth with a dramatic increase of volume in mammals $[4,19,24]$. The organogenesis of the lung in mice can be divided into six different stages: embryonic (embryonic days [E] 9.5-12), pseudoglandular (E12-E16.5), canalicular (E16.5-E17.5), saccular (E17.5-postnatal days [P] 4) and alveolar stage (P4P28) and stage of vascular maturation [19]. Furthermore, alveolarization in mice can be subdivided into two phases; phase one (days 4-21) represents the formation of new alveolar septa from immature pre-existing septa, while phase two (day 14-young adulthood/day 36) is characterized by the lifting off of new alveolar septa from mature pre-existing septa [21].

In the present study, we observed a gradual increase of PLD level in lungs until postnatal week 4. It is suggested that PLD is associated with formation of septa for pulmonary alveolarization because the time point in this study closely matched with the stage of septa formation in mice, as shown previously $[17,19,21]$. This finding is further supported by the finding of a declined PLD level at postnatal 8 week, when vigorous cell proliferation for septa formation was very rare or completely absent in mice. The expression of PLD isozymes PLD1 and PLD2 during postnatal development of lung showed a similar pattern, although the level of PLD1 was significantly expressed during development compared with that of PLD2. Therefore, it is speculated that PLD1 might be a major PLD isozyme involved in the development of mouse lung.

In addition to the alveolarization in the lung, increase of pneumocytes is recognized in the lungs during postnatal development $[22,23]$. These findings suggest that PLD is 
involved in the postnatal lung development including alveolarization and pneumocyte differentiation. Furthermore, various PLD agonists may exist during organogenesis, possibly stimulating PLD, and consequently, activating downstream signals including Src kinases, p-38 mitogen activated protein kinase, calcium and small $\mathrm{G}$ proteins in pulmonary epithelial and vascular endothelial cells [6].

PLD is also involved in the processes of cellular differentiation and/or apoptosis [18], in which both are two important hallmarks of lung morphogenesis during the first several weeks after birth [20]. In the present study, we found that a variety of cell types including the bronchial, bronchiolar, and alveolar epithelial cells and macrophages contained PLD in all lung tissues examined (postnatal weeks 1-8). Although we have not clearly discriminated against the cell phenotype of PLD immunoreactivity during lung development, it is possible that the majority of PLD positive cells are proliferative, while some cells are apoptotic leading to the thinning of the alveolar septa [20]. However, we postulate that the number of PLD associated apoptotic cells are very few, if any, at postnatal 8 week, because the level of PLD was similarly low to the level of postnatal week 1. We also postulate that PLD is involved in the apoptotic elimination of cells during early lung development, partly for the thinning of alveolar septa. The precise relationship between PLD activation and apoptosis in lung development remains to be further studied.

Taken all the data into consideration, the present study shows that PLD immunoreactivity is transiently increased in mouse lungs until postnatal week 4 , at which time it is partly matched with formation of septa for alveolarization.

ACKNOWLEDGEMENT. This work was supported by a program of the Basic Atomic Energy Research Institute (BAERI), a part of the Nuclear R\&D Programs funded by the Ministry of Science \& Technology (MOST) of Korea.

\section{REFERENCES}

1. Alejandre-Alcázar, M. A., Shalamanov, P. D., Amarie, O. V., Sevilla-Pérez, J., Seeger, W., Eickelberg, O. and Morty, R. E. 2007. Temporal and spatial regulation of bone morphogenetic protein signaling in late lung development. Dev. Dyn. 236: $2825-2835$.

2. Amy, R. W., Bowes, D., Burri, P. H., Haines, J. and Thurlbeck, W. M. 1977. Postnatal growth of the mouse lung. J. Anat. 124: 131-151.

3. Boarder, M. R. 1994. A role for phospholipase D in control of mitogenesis. Trends Pharmacol. Sci. 15: 57-62.

4. Burri, P. H. 1974. The postnatal growth of the rat lung. 3. Morphology. Anat. Rec. 180: 77-98.

5. Copland, I. and Post, M. 2004. Lung development and fetal lung growth. Paediatr. Respir. Rev. 5 (Suppl. A): S259-S264.

6. Cummings, R., Parinandi, N., Wang, L., Usatyuk, P. and Natarajan, V. 2002. Phospholipase D/phosphatidic acid signal transduction: role and physiological significance in lung. Mol. Cell. Biochem. 234/235: 99-109.
7. Exton, J. H. 1998. Phospholipase D. Biochim. Biophys. Acta. 1436: $105-115$.

8. Flint, F. F., Schulte, B. A. and Spicer, S. S. 1986. Glycoconjugate with terminal alpha galactose. A property common to basal cells and a subpopulation of columnar cells of numerous epithelia in mouse and rat. Histochemistry 84: 387-395.

9. Judd, W. J., Murphy, L. A., Goldstein, I. J., Campbell, L. and Nichols, M. E. 1978. An anti-B reagent prepared from the alpha-D-galactopyranosyl-binding isolectins from Bandeiraea simplicifolia seeds. Transfusion 18: 274-280.

10. Kim, H., Kang, T. Y., Joo, H. G. and Shin, T. 2006. Immunohistochemical localization of galectin-3 in boar testis and epididymis. Acta Histochem. 108: 481-485.

11. Kim, H., Lee, J., Kim, S., Shin, M. K., Min, D. S. and Shin, T. 2007. Differential expression of phospholipases D1 and D2 in mouse tissues. Cell Biol. Int. 31: 148-155.

12. Kim, M., Moon, C., Kim, H., Shin, M. K., Min, D. S. and Shin, T. 2009. Developmental levels of phospholipase D isozymes in the brain of developing rats. Acta Histochem. (in press).

13. Kim, S., Kim, H., Lee, Y., Hyun, J. W., Lee, Y. H., Shin, M. K., Min, D. S. and Shin, T. 2007. The expression and cellular localization of phospholipase D isozymes in the developing mouse testis. J. Vet. Sci. 8: 209-212.

14. Lee, E. J., Min, D. S., Lee, M. Y., Chung, J. W., Chun, M. H. and Oh, S. J. 2002. Differential expression of phospholipase D1 in the developing retina. Eur. J. Neurosci. 15: 1006-1012.

15. Min, D. S., Choi, J. S., Chun, M. H., Chung, J. W. and Lee, M. Y. 2001. Transient expression of phospholipase D1 in developing rat hippocampus. Neurosci. Lett. 310: 125-128.

16. Moon, C., Kim, H., Kim, S., Lee, Y., Shin, M. K., Min, D. S. and Shin, T. 2008. Transient expression of phospholipase D1 during heart development in rats. J. Vet. Med. Sci. 70: 411413.

17. Mund, S. I., Stampanoni, M. and Schittny, J. C. 2008. Developmental alveolarization of the mouse lung. Dev. Dyn. 237: 2108-2116.

18. Nakashima, S. and Nozawa, Y. 1999. Possible role of phospholipase D in cellular differentiation and apoptosis. Chem. Phys. Lipids 98: 153-164.

19. Roth-Kleiner, M. and Post, M. 2005. Similarities and dissimilarities of branching and septation during lung development. Pediatr. Pulmonol. 40: 113-134.

20. Schittny, J. C., Djonov, V., Fine, A. and Burri, P. H. 1998. Programmed cell death contributes to postnatal lung development. Am. J. Respir. Cell Mol. Biol. 18: 786-793.

21. Schittny, J. C., Mund, S. I. and Stampanoni, M. 2008. Evidence and structural mechanism for late lung alveolarization. Am. J. Physiol. Lung Cell Mol. Physiol. 294: L246-L254.

22. Schmiedl, A., Ochs, M., Muhlfeld, C., Johnen G. and Brasch, F. 2005. Distribution of surfactant proteins in type II pneumocytes of newborn, 14-day old, and adult rats: an immunoelectron microscopic and stereological study. Histochem. Cell Biol. 124: 465-476.

23. Schmiedl, A., Vieten, G., Muhlfeld, C. and Bernhard, W. 2007. Distribution of intracellular and secreted surfactant during postnatal rat lung development. Pediatr. Pulmonol. 42: 548562.

24. Vidic, B. and Burri, P. H. 1983. Morphometric analysis of the remodeling of the rat pulmonary epithelium during early postnatal development. Anat. Rec. 207: 317-324. 